\title{
Direct Measurement of the Torsional Rigidity of Single Actin Filaments
}

\section{Ryohei Yasuda, Hidetake Miyata* and Kazuhiko Kinosita, Jr}

\author{
Department of Physics \\ Faculty of Science and \\ Technology, Keio University \\ Hiyoshi 3-14-1, Kohoku-ku \\ Yokohama 223, Japan
}

\begin{abstract}
Flexural and torsional rigidities of actin filaments are important factors in cell motility and muscle contraction, where actin filaments serve as mechanical elements. The flexural rigidity has already been determined by directly observing the bending of individual filaments under a microscope, but measurement of the torsional rigidity has been relatively scarce and indirect, because torsion of an actin filament is difficult to visualize. This paper shows that the torsional rigidity can be measured directly by visualizing the torsional Brownian motion of a single actin filament with a novel methodology based on an optical trapping technique. Actin filaments (F-actin) were prepared by polymerizing actin monomers binding $\mathrm{Ca}^{2+}$ ion or $\mathrm{Mg}^{2+}$ ion at the high affinity site. The torsional rigidity of F-Ca ${ }^{2+}$-actin $\left((8.5( \pm 1.3)) \times 10^{-26} \mathrm{~N} \mathrm{~m}^{2}\right)$ was about three times as large as that of F-Mge ${ }^{2+}$-actin $\left((2.8( \pm 0.3)) \times 10^{-26} \mathrm{~N} \mathrm{~m}^{2}\right)$, whereas the flexural rigidity $\left((6.0( \pm 0.2)) \times 10^{-26} \mathrm{~N} \mathrm{~m}^{2}\right)$ was almost independent of the kind of the bound cation. The dynamic structure of F-actin is regulated by the bound metal in an anisotropic manner. The torsional rigidities above, whether of $\mathrm{F}-\mathrm{Ca}^{2+}$-actin or $\mathrm{F}-\mathrm{Mg}^{2+}$-actin, are one to two orders of magnitude greater than previous experimental estimates.
\end{abstract}

(C) 1996 Academic Press Limited

Keywords: flexural rigidity; divalent cation; optical tweezers; optical microscope; fluorescence microscope (Yanagida et al., 1984; Ott et al., 1993; Gittes et al., 1993; Isambert et al., 1995), suggesting that the structure of an actin filament cannot be regarded as a homogenous isotropic rod. Theoretically, however, such a large difference between torsional and flexural rigidities is not readily compatible with the helical structure of F-actin (Erickson, 1989). Theoretical estimates based on atomic models of F-actin (ben-Avraham \& Tirion, 1995) suggest that the torsional rigidity is of the same order of magnitude as the flexural rigidity. Resolution calls for experiments that bear more unambiguously on the filament torsion.

In this paper, we describe a system that allows direct visualization of the torsional motion of a single actin filament under an optical microscope. Because the dynamic properties of F-actin have been suggested to depend on the species of tightly bound divalent cation (Mihashi et al., 1979; Orlova \& Egelman, 1993), we compared the torsional and flexural rigidities of $\mathrm{F}-\mathrm{Ca}^{2+}$-actin and $\mathrm{F}-\mathrm{Mg}^{2+}$-actin. In both types of F-actin, the torsional rigidity was of the same order of magnitude as the flexural rigidity, basically in agreement with the theories. Quantitatively, however, the torsional rigidity of 
$\mathrm{F}-\mathrm{Ca}^{2+}$-actin was found to be three times larger than that of $\mathrm{F}-\mathrm{Mg}^{2+}$-actin. In contrast, the flexural rigidity did not depend on the species of the bound divalent cation. The cation introduces a highly anisotropic response in the mechanical properties of F-actin.

\section{Results}

\section{Torsional motion of a single actin filament visualized with a bead duplex}

Axial rotation of an actin filament with a diameter of about $10 \mathrm{~nm}$ (Holmes et al., 1990) cannot be directly resolved by optical microscopy. Thus we attached to an actin filament, with optical tweezers, a duplex of polystyrene beads as a marker of the filament rotation. The ends of the filament were bound to separate beads fixed on the bottom surface of a flow chamber (Figure 1(a) to (c)). The positions of the upper and lower beads in the duplex were clearly resolved in the bright-field image (Figure 1(c)). The duplex changed its orientation with time, as shown in Figure 1(d). It was an irregular to and fro motion, indicating thermal origin. Traces (projection onto a horizontal, or $x-y$, plane) of the movement of the upper and lower beads (Figure 1(e)) show that the irregular motion of the bead duplex reflected primarily the torsional Brownian motion of the actin filament: both traces are perpendicular to the filament axis (taken as $y$-axis), and the excursion of the upper bead is larger than that of the lower bead (by a factor of about three in Figure 1(e), as expected). Before analyzing the torsional motion in detail, we examine possible contributions from other types of motion.

Bending Brownian motion of the filament will also move the bead duplex. Small amplitude bending in the $x-y$ plane will result in translation, without reorientation, of the bead duplex in the $x$-direction. This was observed in many samples: the $x$-excursion (measured as standard deviation) of the upper bead was often only twice that of the lower bead, and in extreme cases the ratio was as low as 1.5. Because this ratio should be equal to, or greater than, three for pure torsion (the ratio exceeds three when the filament makes extensive contact with the lower bead and is wound around the bead), the low ratio is ascribed to bending. The amplitude of the bending motion, however, was small, because we selected those actin filaments that appeared straight between the end beads. The $x$-excursion of the lower bead, which is an upper limit of the bending motion, was at most $( \pm 0.3) \mu \mathrm{m}$ (standard deviation), and the ratio of the size of $x$-excursion to the filament length did not exceed 0.05. In some cases the bending was also apparent in the fluorescence image of the filament.

The bending motion in the $x-y$ plane is cancelled in Figure 1(f) which shows the trace of the motion of the upper bead relative to the lower bead. This trace reflects changes in the tilt of the bead duplex. The spread of this trace along the $y$-direction is close to the precision of the measurement $(( \pm 1)$

(a)

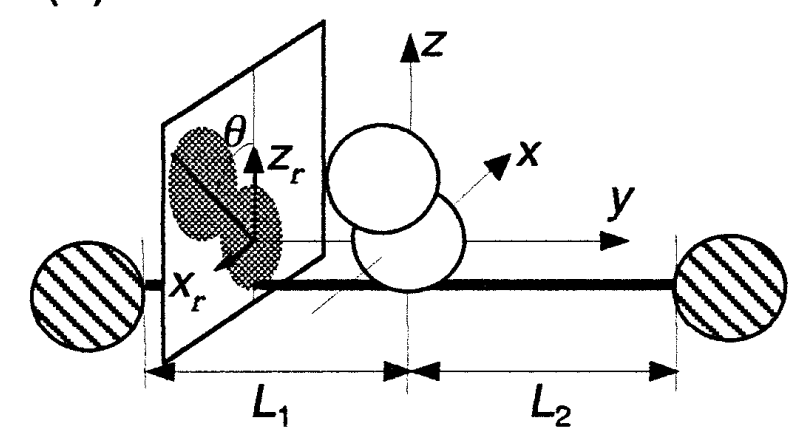

(b)

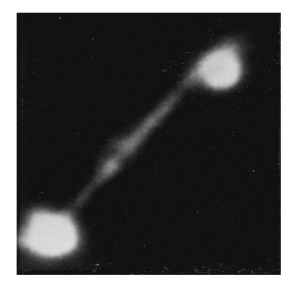

(c)

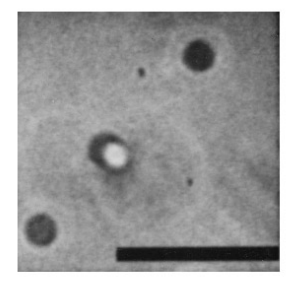

(d)

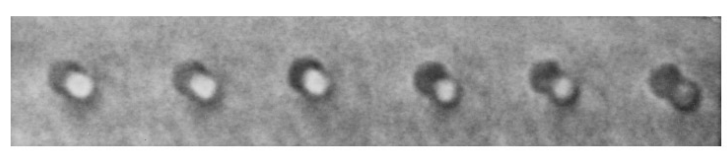

(e)
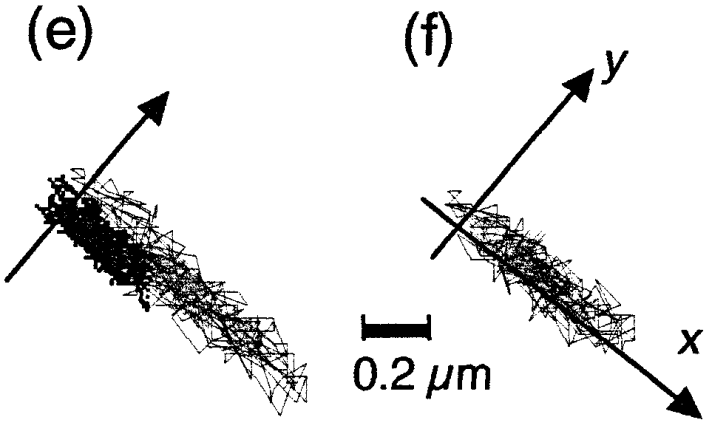

Figure 1. Direct observation of the torsional Brownian motion of an actin filament. (a) Schematic illustration. The striped beads are fixed on a surface (the surface is not shown). $x$ and $y$ axes are respectively perpendicular and parallel to the filament axis in the horizontal (image) plane, and $z$ is a vertical axis. $x_{\mathrm{r}}$ and $z_{\mathrm{r}}$ are the coordinates of the upper bead relative to the lower bead. $\theta$ is the tilt angle of the bead duplex in the $x-z$ plane. (b) Fluorescence image (the bead duplex at the center is out of focus). (c) Bright-field image. The microscope focus was adjusted so that the upper bead appeared brighter. Bar, $5 \mu \mathrm{m}$. (d) Snapshots of the fluctuating bead duplex at intervals of $0.13 \mathrm{~s}$. The tilt angle, $\theta$, in these images was determined as $37^{\circ}, 36^{\circ}, 27^{\circ}, 47^{\circ}, 50^{\circ}$, and $53^{\circ}$. (e) Motions of the upper (thin line) and lower (thick line) beads in the duplex projected onto the $x-y$ plane. $y$-axis is parallel to the filament axis. Bead coordinates were read by fitting a circle to the bead image by eye. (f) Motion of the upper bead relative to the lower bead, projected onto the $x-y$ plane. The relative coordinates $\left(x_{\mathrm{r}}, y_{\mathrm{r}}\right)$ were calculated as $\left(x_{u}-x_{l}, y_{u}-y_{l}\right)$ where $\left(x_{u}, y_{u}\right)$ and $\left(x_{l}, y_{l}\right)$ are, respectively, the coordinates of the upper and lower beads in (e). 

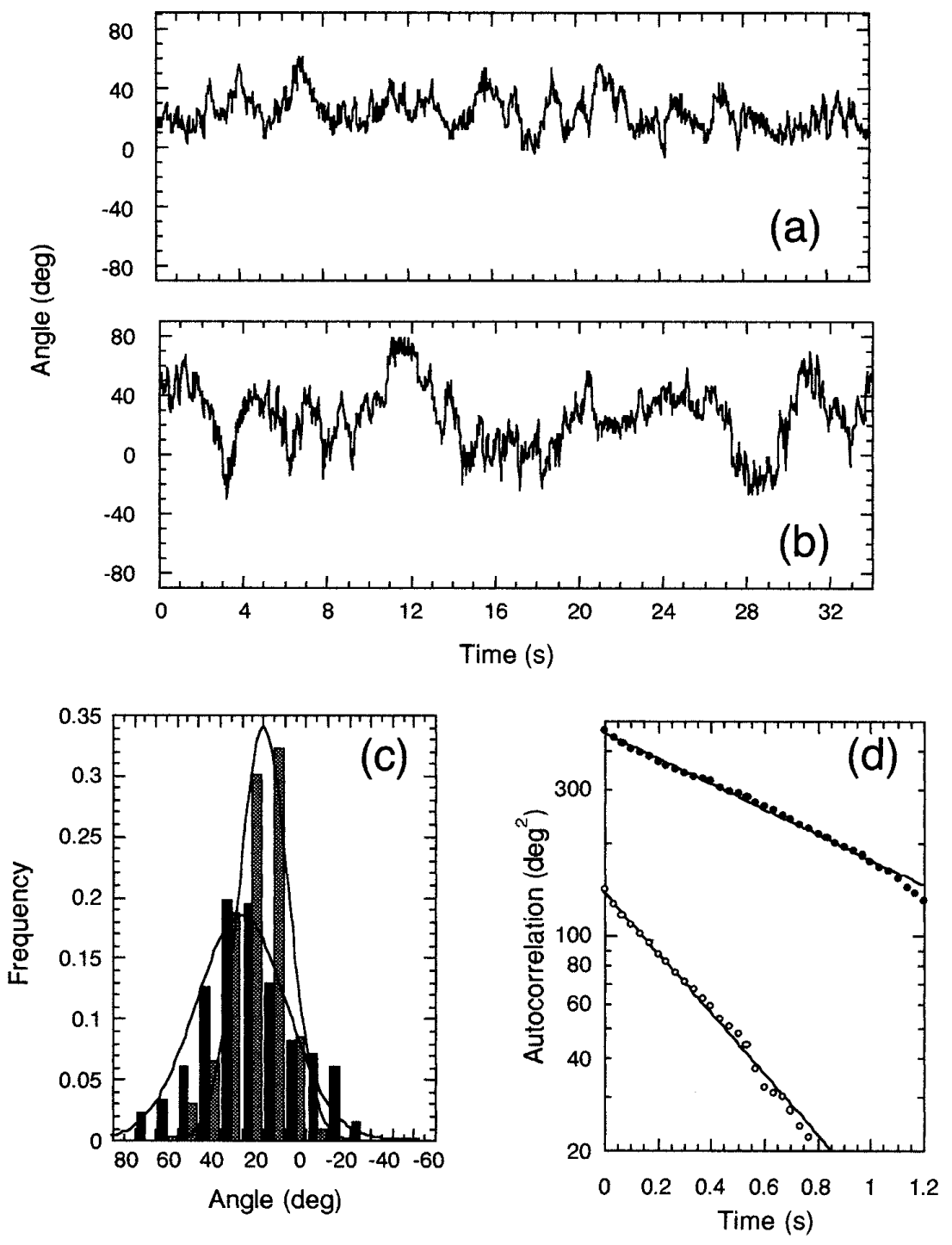

Figure 2. (a) and (b), Fluctuation of the rotational angle, $\theta$, of a bead duplex sampled at intervals of $33 \mathrm{~ms}$ (video rate). In (a) the duplex was bound to a $\mathrm{Ca}^{2+}$-actin filament at $L_{1}=3.5 \mu \mathrm{m}$ and $L_{2}=2.8 \mu \mathrm{m}$ (see Figure 1(a)), and in (b) to a $\mathrm{Mg}^{2+}$-actin filament at $L_{1}=2.9 \mu \mathrm{m}$ and $L_{2}=2.4 \mu \mathrm{m}$. (c) Distribution of $\theta$ for the $\mathrm{F}_{-} \mathrm{Ca}^{2+}$-actin in (a) (hatched bars) and for $\mathrm{F}-\mathrm{Mg}^{2+}$-actin in (b) (filled bars). Continuous lines indicate a Gaussian fit to each distribution. Variance of the angular distribution, $\left\langle(\theta-\langle\theta\rangle)^{2}\right\rangle$, was $142 \mathrm{deg}^{2}$ for the $\mathrm{F}-\mathrm{Ca}^{2+}$-actin and $470 \mathrm{deg}^{2}$ for the $\mathrm{F}-\mathrm{Mg}^{2}$-actin. To estimate the reliability of these values, the data in (a) and (b) were divided into halves; the first and second halves respectively gave 139 and $127 \mathrm{deg}^{2}$ for the F-Ca ${ }^{2+}$-actin, and 496 and $427 \mathrm{deg}^{2}$ for the F-Mg ${ }^{2+}$-actin. (d) Autocorrelation function of the data in (a) and (b). Continuous lines show a theoretical fit with $\exp (-t / \tau)$, where $\tau=0.42 \mathrm{~s}$ for the $\mathrm{F}-\mathrm{Ca}^{2+}$-actin (open circles) and $\tau=1.0 \mathrm{~s}$ for the $\mathrm{F}-\mathrm{Mg}^{2+}$-actin (filled circles). Division of (a) and (b) into halves yielded $\tau=0.50$ and $0.33 \mathrm{~s}$ for the $\mathrm{F}_{-\mathrm{Ca}^{2+} \text {-actin and }}$ $\tau=0.97$ and $0.91 \mathrm{~s}$ for the $\mathrm{F}-\mathrm{Mg}^{2}$ actin.

pixel or $( \pm 0.04) \mu \mathrm{m})$. Bending in the $y-z$ plane also contributes to the $y$-spread, but the vertical bending was negligible in this sample as was also the case for horizontal bending (as indicated by the approximately $3: 1$ ratio in the $x$-excursions of the upper and lower beads in Figure 1(e)). On average, the relative motion in $y$-direction was characterized by a standard deviation of $( \pm 0.1) \mu \mathrm{m}$, corresponding to tilting in the $y-z$ plane of $\left( \pm 7^{\circ}\right)$.

The bead duplex was glued to the actin filament via $\alpha$-actinin. The small $y$-excursion in Figure $1(\mathrm{f})$ suggests that the linkage was rigid and did not allow independent fluctuation of the bead duplex on the filament, as was also the case for a bead duplex bound to F-actin with gelsolin (Suzuki et al., 1996). The tilting in the $y-z$ plane of $\left( \pm 7^{\circ}\right)$ above sets an upper limit for the amplitude of the independent fluctuation (in the $y$-direction).

\section{Derivation of the torsional rigidity}

Analysis of the torsional motion proceeds as follows. The tilt angle $\theta$ in the $x-z$ plane (Fig- ure 1(a)) is calculated from the relative coordinates $\left(x_{r}, y_{r}\right)=\left(x_{u}-x_{l}, y_{u}-y_{l}\right)$, where $\left(x_{u}, y_{u}\right)$ and $\left(x_{l}, y_{l}\right)$ are the coordinates of the upper and lower beads:

$$
\theta=\arctan \left(x_{r} / z_{r}\right)=\arctan \left\{x_{r} /\left(d^{2}-y_{r}^{2}-x_{r}^{2}\right)^{1 / 2}\right\}
$$

where $d=0.88 \mu \mathrm{m}$ is the distance between the centers of the two beads, and $z_{r}=\left(d^{2}-y_{r}^{2}-x_{r}^{2}\right)^{1 / 2}$ is the relative coordinate in the $z$-direction. The error $\Delta \theta$ in $\theta$ above is estimated as $\Delta \theta \sim(\Delta x / d) / \cos \theta$, where $\Delta x \sim 0.04 \mu \mathrm{m}$ ( $=1$ pixel) is the precision of $x ; \Delta \theta \sim 2.6^{\circ}$ for $\theta=0^{\circ}, \Delta \theta \sim 5^{\circ}$ for $\theta \sim\left( \pm 60^{\circ}\right)$, and $\Delta \theta \sim 15^{\circ}$ for $\theta=\left( \pm 80^{\circ}\right)$. To avoid larger error, samples in which $\theta$ exceeded $\left( \pm 80^{\circ}\right)$ were not subjected to analysis.

Figure 2(a) and (b) show typical time courses of the fluctuation of $\theta$. The amplitude of the fluctuation is clearly different between the two plots, as is also evident in Figure 2(c) which shows the distribution of $\theta$ values. Figure 2(a) represents torsional fluctuation of a filament made of actin with $\mathrm{Ca}^{2+}$ at the tightly binding site and Figure 2(b) a filament of $\mathrm{Mg}^{2+}$ actin with a similar length. Apparently, the torsional spring that confined the 
fluctuation of the bead duplex was stiffer in the $\mathrm{Ca}^{2+}$-actin filament $\left(\mathrm{F}^{-\mathrm{Ca}^{2+}}\right.$-actin) than in the $\mathrm{Mg}^{2+}$-actin filament (F-Mg ${ }^{2+}$-actin).

The spring constant, $k$, can be estimated from the variance of the angular distribution, $\left\langle(\theta-\langle\theta\rangle)^{2}\right\rangle$ where \langle\rangle represent the average over time, using the equipartition principle: $k=k_{B} T /\left\langle(\theta-\langle\theta\rangle)^{2}\right\rangle$, where $k_{\mathrm{B}} T$ is the thermal energy (Wang \& Uhlenbeck, 1954). For the $\mathrm{F}_{-\mathrm{Ca}^{2+}}$-actin in Figure 2(a), $k$ was obtained as $9.4 \times 10^{-20} \mathrm{~N} \mathrm{~m}$, and for the F- $\mathrm{Mg}^{2+}$-actin in Figure 2(b), $k$ was $2.9 \times 10^{-20} \mathrm{~N} \mathrm{~m}$. Because of the limited time resolution in the video analysis, the variance was underestimated, hence $k$ was overestimated, by 1 to 3\% (see equation (6) in Materials and Methods).

Alternatively, the torsional spring constant, $k$, can also be obtained from an autocorrelation analysis of the angular fluctuation data (Wang \& Uhlenbeck, 1954). The autocorrelation function, $\langle\{\theta(t)-\langle\theta\rangle\}\{\theta(t+s)-\langle\theta\rangle\}\rangle$ where $t$ and $s$ are time, could be fitted with an exponential (Figure 2(d)), as expected for the rotational Brownian motion restricted by a torsional spring. The spring constant, $k$, is related to the correlation time $\tau$ by an equation $k=\xi / \tau$, where $\xi$ is the frictional coefficient. For the rotation of a bead duplex around the actin filament attached at the bottom of the duplex (Figure 1(a)), $\xi$ is given as:

$$
\begin{aligned}
\xi=C_{1} 8 \pi \eta r^{3}+C_{2} 6 \pi \eta r & (3 r)^{2} \\
& +C_{3} 8 \pi \eta r^{3}+C_{4} 6 \pi \eta r(r)^{2}
\end{aligned}
$$

where $r=0.44 \mu \mathrm{m}$ is the radius of the beads, and $\eta=0.93 \times 10^{-3} \mathrm{~Pa} \mathrm{~s}$ is the bulk viscosity of water at $23^{\circ} \mathrm{C}$. $C_{1}$ through $C_{4}$ are numerical factors that account for higher viscous drag in proximity to the glass surface than in the bulk water. The first and third terms on the right-hand side derive from the rotation of each bead around is center, and the second and fourth from the translation with respect to the central filament; hydrodynamic interaction between the beads is neglected. $C_{1}$ and $C_{2}$ are the correction factors for the rotation and translation, respectively, of the upper bead with its center at the height of $4 r$ measured from the glass surface, and $C_{3}$ and $C_{4}$ are those for the lower bead at $2 r$ (Figure 1(a)). According to Goldman et al. (1967), $C_{1}$ through $C_{4}$ for the above geometry are given as 1.0, 1.2, 1.0 and 1.4. The friction coefficient $\xi$ is thus calculated as $2.3 \times 10^{-20} \mathrm{~N} \mathrm{~m} \mathrm{~s}$. From the correlation times obtained in Figure 2(d), the spring constant, $k$, was estimated as $5.5 \times 10^{-20} \mathrm{~N} \mathrm{~m}$ for the

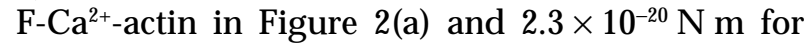
the F- $\mathrm{Mg}^{2+}$-actin in Figure 2(b). These values agree fairly well with the estimation from the equipartition principle above. Precise agreement cannot be expected, in view of the uncertainties involved in the estimation of $\xi$ (e.g. $\xi$ will be greater if the beads were closer to the glass surface). In the rest of this paper, we adopt the values obtained from the equipartition principle, which does not involve uncertain parameters.

From the spring constant, $k$, we calculate the

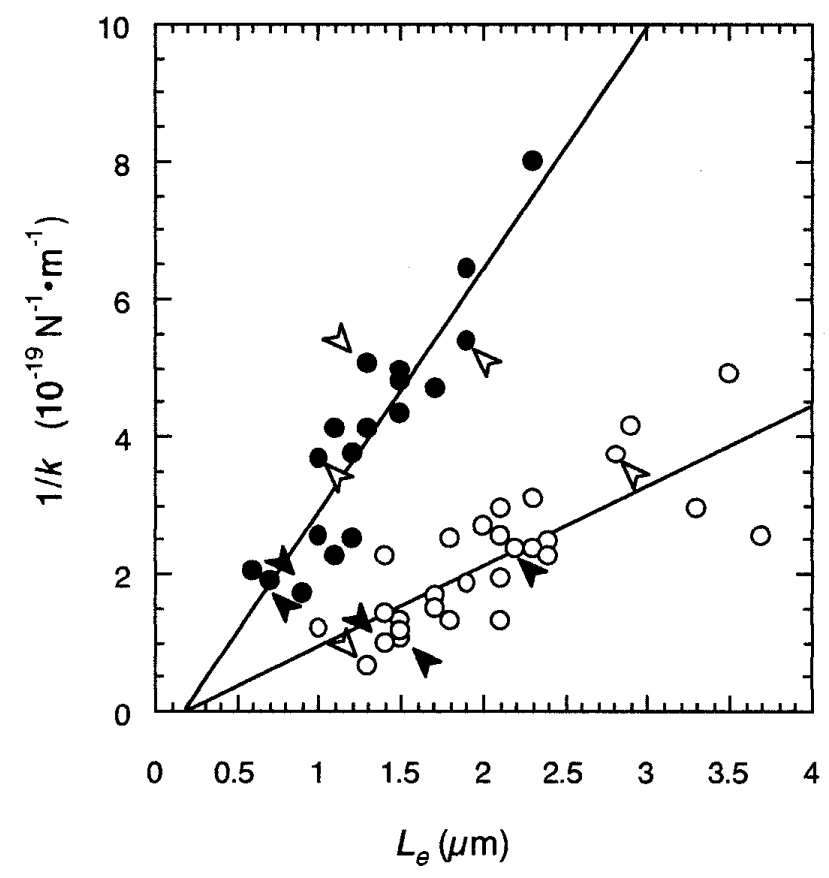

Figure 3. Reciprocal of the spring constant, $1 / k$, versus the effective length, $L_{e}$, of F-Ca ${ }^{2+}(O)$ and $\mathrm{F}_{-} \mathrm{Mg}^{2+}$-actin (O). Each point represents one filament, from which 64 values of $\theta$ were measured at intervals of 0.53 seconds (total 34 seconds). Points with a white arrowhead show filaments under a tension of at least 1.5 to $1.8 \mathrm{pN}$, and points with a black arrowhead under a tension of at most 0.2 to $0.3 \mathrm{pN}$ (see Materials and Methods). Continuous lines show linear regression lines. Samples in which $\theta$ exceeded $\left( \pm 80^{\circ}\right)$ or $\theta$ showed a slow drift over several seconds (presumably due to medium flow) were discarded. In the calculation of $L_{e}$, the lengths $L_{1}$ and $L_{2}$ were taken as in Figure 1(a). These lengths are overestimates if the filament was wound around the lower bead and are underestimates if the attachment to an end bead was at a further point. The errors are, at most, $0.44 \mu \mathrm{m}$ (the radius of the beads), and thus the error in $L_{e}$ is $\sim 0.2 \mu \mathrm{m}$.

torsional rigidity $\kappa_{T}$ in the following way. In our system, two parts of the filament, with lengths $L_{1}$ and $L_{2}$, held the duplex of beads (Figure 1(a)). The corresponding spring constants, $k_{1}$ and $k_{2}$, are related to the torsional rigidity $\kappa_{T}$ of $\mathrm{F}$-actin by $k_{n} L_{n}=\kappa_{T}(n=1,2)$ (Landau \& Lifshitz, 1970). The overall spring constant, $k=k_{1}+k_{2}$, is thus given by:

$$
k=\kappa_{T}\left(1 / L_{1}+1 / L_{2}\right)=\kappa_{T} / L_{e}
$$

where $L_{e}=L_{1} L_{2} /\left(L_{1}+L_{2}\right)$ is an effective length of the spring. Figure 3 shows that experimental $1 / k$ values were proportional to $L_{e}$ for both $\mathrm{F}-\mathrm{Ca}^{2+}$ - and $\mathrm{F}-\mathrm{Mg}^{2+}$-actin, as expected.

\section{Comparison of the elastic properties of $\mathrm{F}-\mathrm{Ca}^{2+}$-actin and $\mathrm{F}-\mathrm{Mg}^{2+}$-actin}

We compared the torsional and flexural rigidities of $\mathrm{F}_{-} \mathrm{Ca}^{2+}$ and $\mathrm{F}-\mathrm{Mg}^{2+}$-actin. All experiments were carried out under the same ionic conditions. Hence, we presume that the only difference between 
$\mathrm{F}^{-} \mathrm{Ca}^{2+}$ and $\mathrm{F}-\mathrm{Mg}^{2+}$-actin is the metal ion at the tightly binding site.

\section{Torsional rigidity}

In Figure 3, the reciprocal of the torsional spring constant, $1 / k$, is plotted against the effective length of the filament, $L_{e}$, for 28 filaments of $\mathrm{Ca}^{2+}$-actin (open circles) and 18 filaments of $\mathrm{Mg}^{2+}$-actin (filled circles). Continuous lines show the result of linear regression analysis for each data set. The intercept at the abscissa is $0.21 \mu \mathrm{m}$ for $\mathrm{F}^{-C^{2+}}{ }^{2+}$-actin with a standard error of $0.30 \mu \mathrm{m}$, and $0.18( \pm 0.13) \mu \mathrm{m}$ for $\mathrm{F}-\mathrm{Mg}^{2+}$-actin. These values are essentially zero, when the error due to the scatter and the uncertainty in $L_{e}$ of $\sim 0.2 \mu \mathrm{m}$ is taken into account. Thus, the data are in accord with equation (3) above. The slope of the regression line gives the torsional rigidity, $\kappa_{T}$, which was $(8.5( \pm 1.3)) \times$

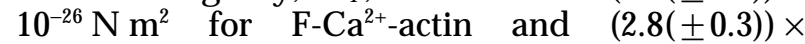

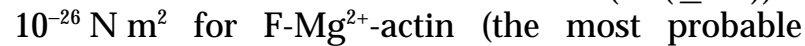
value ( \pm standard error)). $\mathrm{F}-\mathrm{Ca}^{2+}$-actin was more rigid than $\mathrm{F}-\mathrm{Mg}^{2+}$-actin by a factor of approximately three.

The actin filaments in our system were slightly under tension, as a result of the bending Brownian motion between the fixed ends. The magnitude of the tension was estimated from the amplitude of the bending motion (equation (9) in Materials and Methods). In Figure 3, points with a white arrowhead show filaments under a tension of at least 1.5 to $1.8 \mathrm{pN}$, and those with a black arrowhead under a tension of, at most, 0.2 to $0.3 \mathrm{pN}$. A loose filament of $\mathrm{Ca}^{2+}$-actin under a tension less than $0.05 \mathrm{pN}$ gave $\kappa_{\mathrm{T}}$ of $7.3 \times 10^{-26} \mathrm{~N} \mathrm{~m}^{2}$ (not included in Figure 3). Tension up to a few piconewton does not appear to affect the torsional rigidity.

\section{Flexural rigidity}

The flexural rigidity was estimated from the shape of single actin filaments undergoing bending Brownian motion, as in Ott et al. (1993) and Isambert et al. (1995). To continuously observe the whole part of an actin filament, F-actin was confined in a thin cell ( 1 to $2 \mu \mathrm{m}$ thick) where filaments underwent two-dimensional Brownian motion. The flexural rigidity, $\kappa_{F}$, was calculated from CCF (cosine correlation function) as:

$$
\mathrm{CCF}(L)=\langle\boldsymbol{t}(x) \boldsymbol{t}(x+L)\rangle=\exp \left(-L k_{B} T / 2 \kappa_{F}\right)
$$

where $L$ and $x$ are distances along the filament and $t$ is a unit vector along the tangent of the filament.

Semi-log plots of CCF versus L are shown in Figure 4 . The filament rigidity was obtained from the slope of the plot as $(5.8( \pm 0.1)) \times 10^{-26} \mathrm{~N} \mathrm{~m}^{2}$ for F-Ca ${ }^{2+}$-actin and $(6.2( \pm 0.1)) \times 10^{-26} \mathrm{~N} \mathrm{~m}^{2}$ for F- $\mathrm{Mg}^{2+}$ actin. In sharp contrast to the torsional rigidity above, the magnitude of the flexural rigidity did not depend on the species of the tightly bound divalent cation. The above values agree with other solution results on phalloidin-stabilized actin

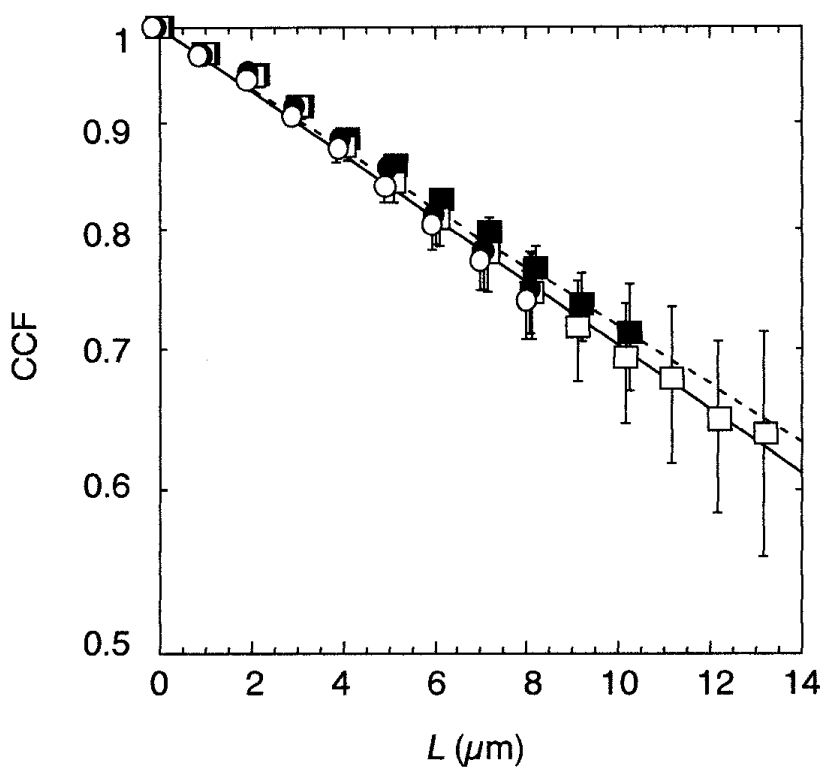

Figure 4. CCF for the bending Brownian motion of

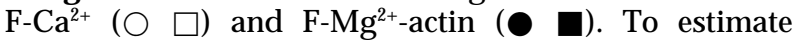
reliability of the measurement, filaments 9 to $10 \mu \mathrm{m}(\bigcirc$ -) and 11 to $19 \mu \mathrm{m}$ ( $\square \square$ ) long were analyzed separately. Each symbol shows the average over 112 to 128 images ( 7 or 8 filaments), the bar being standard error ( $n=7$ or 8$)$. CCF for each symbol was fitted with the function $\exp \left(-L k_{B} T / 2 \kappa_{F}\right)$, giving the flexural rigidity, $\kappa_{F}$, of $(5.77(\mathrm{O})$ and $5.84(\square)) \times 10^{-26} \mathrm{~N} \mathrm{~m}^{2}$ for $\mathrm{F}^{-\mathrm{Ca}^{2+}}$-actin and $(6.10(\mathbf{O})$ and $6.35(\boldsymbol{\square})) \times 10^{-26} \mathrm{~N} \mathrm{~m}^{2}$ for F-Mg $\mathrm{Mg}^{2+}$-actin. From the two values of $\kappa_{F}$ in the same type of actin, the accuracy was estimated as $\sim 0.1 \times 10^{-26} \mathrm{~N} \mathrm{~m}^{2}$. Lines indicate the averages of the two exponential functions, continuous line for $\mathrm{F}_{-} \mathrm{Ca}^{2+}$-actin and broken line for $\mathrm{F}^{-\mathrm{Mg}^{2+} \text {-actin. }}$

(Yanagida et al., 1984; Gittes et al., 1993; Ott et al., 1993; Isambert et al., 1995) of $(6.5-7.4) \times 10^{-26} \mathrm{~N} \mathrm{~m}^{2}$ as well as the theoretical estimate (ben-Avraham \& Tirion, 1995) of $(4.3-5.8) \times 10^{-26} \mathrm{~N} \mathrm{~m}^{2}$.

\section{Discussion}

\section{Comparison with previous studies}

We have measured the torsional and flexural rigidities of $\mathrm{F}_{-} \mathrm{Ca}^{2+}$ and $\mathrm{F}-\mathrm{Mg}^{2+}$-actin. The torsional rigidity was of the same order of magnitude as the flexural rigidity for both types of actin. In this sense, the elastic properties of F-actin on the scale of micrometers are not very different from those of a homogenous isotropic rod. However, experimental work (Egelman et al., 1982; Egelman \& DeRosier, 1992; Yoshimura et al., 1984; Prochniewicz et al., 1996) has indicated a torsional rigidity one to two orders of magnitude smaller than the flexural rigidity. Thus, a torsional angular disorder $\Delta \phi$ of $5^{\circ}$ to $6^{\circ}$ between two consecutive actin protomers in a filament has been suggested from electron microscopic images (Egelman et al., 1982; Egelman \& DeRosier, 1992). This large radial disorder is equivalent to $\kappa_{T}=k_{B} T h /(\Delta \phi)^{2} \sim 0.1 \times 10^{-26} \mathrm{~N} \mathrm{~m}^{2}$, where $h=2.7 \mathrm{~nm}$ is the axial rise per protomer. 
Optical anisotropy decays also gave much smaller $\kappa_{\mathrm{T}}$ than ours: $\sim 0.2 \times 10^{-26} \mathrm{~N} \mathrm{~m}$ for eosinlabeled F- $\mathrm{Mg}^{2+}$-actin in solution (Yoshimura et al., 1984 ), and $0.14 \times 10^{-26} \mathrm{~N} \mathrm{~m}$ for erythrosin-labeled

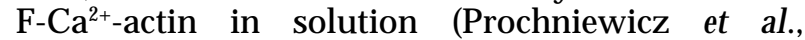
1996). These experimental estimates are difficult to reconcile with our new results. If the radial disorder observed in the electron micrograph is static in nature and persists over many seconds in solution, it might be unnoticed in the analysis of dynamic torsional Brownian motion. Or, staining and/or drying during sample preparation for electron microscopy might have amplified the disorder. In the optical anisotropy decay measurements, labeling with the dye may have introduced an additional flexibility, or else rotation of short filaments might have contributed to the optical signal (Sawyer et al., 1988). With direct imaging, on the other hand, Suzuki et al. (1996) have analyzed the motion of bead aggregates bound to the tail of an actin filament and estimated the torsional rigidity of F-actin to be of a magnitude similar to our values. Tsuda et al. (1996) have also suggested a similar value.

Actin filaments in the present work, as in other optical microscopic work, were stabilized with phalloidin, which might have produced torsionally stiff F-actin. However, we note in the literature, including the work of those groups reporting a very low torsional rigidity, that the effect of phalloidin on properties related to the filament rigidity is, at most, a factor of two: it is unlikely that phalloidin explains the order of magnitude difference between our rigidity values and the previous estimates. Thus, in the optical anisotropy decay measurements, phalloidin did not affect the decay characteristics (Yoshimura et al., 1984) or only slightly (40\%) decreased the torsional rigidity (Prochniewicz et al., 1996). In the electron microscopic analysis (Orlova \& Egelman, 1993), phalloidin did not increase the rigidity when added to normal actin filaments. Bremer et al. (1991) found, in their electron microscopic images, that the distribution of the crossover spacings for the two long-pitch helical strands became narrower upon stabilization with phalloidin, but the difference in the variance was only $50 \%$. Bending Brownian motion in solution indicated that the flexural rigidity was twice higher in the presence of phalloidin than in its absence (Isambert et al., 1995). Motivated by this last work where they succeeded in observing dye-labeled actin filaments without phalloidin as long as 15 minutes (by adding a sufficient amount of unlabeled G-actin), we also attempted to visualize the torsional Brownian motion of unstabilized F-actin by covalent labeling with 5-iodoacetamide-tetramethylrhodamine or iodoacetamide-Cy3. We were, however, unable to finish the time-consuming process of setting up the system in Figure 1(a) before the filament depolymerized in the absence of phalloidin.

\section{Anisotropic dynamics of F-actin}

Mihashi et al. (1979) have found spectroscopic differences between $\mathrm{F}^{-\mathrm{Ca}^{2+}}$-actin and $\mathrm{F}-\mathrm{Mg}^{2+}$-actin and suggested that the tightly bound cation regulates the structure of F-actin in a dual mode. Our results indicate that the structural difference is manifested in the torsional dynamics, but not in bending: the torsional rigidity of $\mathrm{F}_{-} \mathrm{Ca}^{2+}$-actin was $\sim 3$ times larger than that of F- $\mathrm{Mg}^{2+}$-actin (Figure 3), while in contrast, the flexural rigidity did not differ significantly (Figure 4). Somehow, the bound metal elicits a highly anisotropic response in the dynamic structure of F-actin. That the flexural rigidity is insensitive to the bound metal has also been shown by Isambert et al. (1995).

The anisotropic response suggests that F-actin cannot be regarded as a perfectly isotropic rod. In addition our torsional and flexural rigidity values, whether of $\mathrm{F}_{-} \mathrm{Ca}^{2+}$-actin or of $\mathrm{F}-\mathrm{Mg}^{2+}$-actin, do not rigorously conform to the theory for a homogeneous isotropic rod (Landau \& Lifshitz, 1970) which predicts that the ratio of the flexural rigidity to the torsional rigidity should be between 1 and 1.5. The elastic properties of F-actin depend on the details of its atomic structure, as shown by ben-Avraham \& Tirion (1995). Structural determination of $\mathrm{F}^{-\mathrm{Ca}^{2+}}$-actin and $\mathrm{F}-\mathrm{Mg}^{2+}$-actin at high resolution is awaited. On the theoretical side, since the theoretical predictions by ben-Avraham \& Tirion (1995) are close to our results on $\mathrm{F}-\mathrm{Mg}^{2+}-$ actin, it would be interesting to see whether, and what kind of, modifications of the atomic models may lead to a higher torsional rigidity without significant increase in the flexural rigidity.

The dependence of the torsional rigidity on the bound divalent cation suggests that it is possible to assess the species of bound cation through the measurement of the torsional rigidity. For example, the presence of $1 \mathrm{mM} \mathrm{MgCl}{ }_{2}$ during polymerization of $\mathrm{F}_{-} \mathrm{Ca}^{2+}$-actin, as employed in our actin preparation for the in vitro motility assay (Miyata et al., 1995), gave $\kappa_{T}=(7.5( \pm 2.0)) \times 10^{-26} \mathrm{~N} \mathrm{~m}^{2} \quad(n=22)$. This value is close to that of $\mathrm{F}-\mathrm{Ca}^{2+}$-actin, suggesting that the major species of the bound cation in this preparation was $\mathrm{Ca}^{2+}$. This is consistent with the relatively slow exchange of the tightly-bound cation in G-actin and virtually no exchange in F-actin (Estes et al., 1992; Kasai \& Oosawa, 1969; Gershman et al., 1994).

The helical structure of F-actin suggests that its torsional rigidity may depend on the sense of the twist, at least for a large enough deformation. We examined this possibility in three samples in which the bead duplex was off the center $\left(L_{1} / L_{2} \sim 2\right.$; see Figure 1(a)). However, the distribution of the torsional angle, $\theta$, did not show a clear sign of asymmetry above the experimental precision. This is not unexpected, because the deformation by thermal energy is small, the root-mean-square angular deviation between two consecutive protomers in the long-pitch helix being $1.6^{\circ}$ for $\mathrm{F}-\mathrm{Mg}^{2+}$-actin and $1.0^{\circ}$ for $\mathrm{F}_{-} \mathrm{Ca}^{2+}$-actin. A system in 
which only one end of a filament is fixed (e.g. Suzuki et al., 1996; Tsuda et al., 1996) would be slightly better for the detection of anisotropic rigidity, but the best strategy will be to apply a much larger torque with, e.g. optical tweezers.

\section{Torque component in the sliding force between actin and myosin}

Nishizaka et al. (1993) have found a torque component in the sliding force between actin and myosin; an actin filament, of which the front end was fixed to a glass surface, formed a supercoil when the rear part of the filament slid on a track of heavy meromyosin. The magnitude of the torque can now be estimated from the torsional rigidity of F-actin. Because the supercoil would absorb the torsional stress for every complete turn of the rear part, a torque for twisting the $\sim 10 \mu \mathrm{m}$ filament (presumably $\mathrm{Ca}^{2+}$-form) by half a turn, $\pi \kappa_{T} /$ $(10 \mu \mathrm{m}) \sim 30 \mathrm{pN} \mathrm{nm}$, would produce the supercoil. On the other hand, the heavy meromyosin track of length 2 to $3 \mu \mathrm{m}$ would produce a translational sliding force of $\sim 50 \mathrm{pN}$ (T. Nishizaka, unpublished results). The force from the rotational torque, $\sim 6 \mathrm{pN}$ on the surface of F-actin with $5 \mathrm{~nm}$ radius, would thus amount to $\sim 10 \%$ of the sliding force. In a conventional motility assay at a low heavy-meromyosin density, on the other hand, sliding actin filaments were unable to rotate a bead duplex attached at the tail end of the filament (Suzuki et al., 1996). From this observation, Suzuki et al. estimated the rotational component to be less than $10 \%$ of the sliding force. The actomyosin interaction produces a force which operates primarily along the long axis of the actin filament.

In a skeletal muscle, the isometric tension amounts to $230 \mathrm{pN}$ per actin filament (Ford et al., 1981; Kojima et al., 1994). If $10 \%$ of this force produces a torsional torque at the surface of the actin filament, the $1 \mu \mathrm{m}$ filament made of $\mathrm{Mg}^{2+}$ actin (Kitazawa et al., 1982) would be twisted by one turn or so. For a single myosin molecule, which produces an isometric force of 3 to $5 \mathrm{pN}$ (Finer et al., 1994; Ishijima et al., 1994), an actin filament would appear as an almost undeformable wire.

The stiff actin which resists the torque component of the sliding force might be essential for proper operation of a muscle. In a muscle, variable separation between actin and myosin filaments as well as the difference in the helical pitch of the two filaments call for a flexibility in the actomyosin crossbridge (Huxley, 1969). Brownian motion of myosin heads in relaxed myofibrils indicated that the head is linked to the backbone of a myosin filament via at least two flexible joints (Ishiwata et al., 1987). These flexible joints, however, ought to be held taut during efficient force production. A twist of the myosin head around an actin filament, counteracted by the rigid torsional spring of the actin filament, would help strain the slack joints.

\section{Materials and Methods}

\section{Proteins}

Column purified $\mathrm{G}-\mathrm{Ca}^{2+}$-actin was prepared from rabbit skeletal muscle according to Pollard \& Cooper (1982) in $2 \mathrm{mM}$ Tris- $\mathrm{HCl}, 0.2 \mathrm{mM} \mathrm{CaCl}_{2}, 0.2 \mathrm{mM}$ ATP and $0.3 \mathrm{mM} \mathrm{NaN}$. Rabbit skeletal $\alpha$-actinin was prepared according to Suzuki et al. (1976). BSA (bovine serum albumin) was from Sigma Chemicals (St Louis, MO, USA). Concentrations of proteins were estimated from ultraviolet absorption measurements using $A_{280 \mathrm{~nm}}^{1 \%}=9.7$ for $\alpha$-actinin and $A_{290 \mathrm{~nm}}^{1 \%}=6.3$ for G-actin.

Labeled $\mathrm{F}_{-} \mathrm{Ca}^{2+}$-actin was obtained by polymerizing $5 \mu \mathrm{M} \mathrm{G}-\mathrm{Ca}^{2+}$-actin in a standard solution containing $10 \mathrm{mM}$ MOPS-KOH (pH 7.0), 100 mM KCl, 0.07 mM ATP and $10 \mu \mathrm{M}$ phalloidin-tetramethylrhodamine (Fluka, Tokyo, Japan) overnight at $4^{\circ} \mathrm{C}\left(0.07 \mathrm{mM}\right.$ of $\mathrm{CaCl}_{2}$ was carried over from the G-actin solution). Labeled F- $\mathrm{Mg}^{2+}$ actin was obtained as follows: $30 \mu \mathrm{M}$ of G-Ca ${ }^{2+}$-actin was incubated in the solution containing $0.1 \mathrm{mM} \mathrm{MgCl} 2$ and $0.2 \mathrm{mM}$ EGTA ( $\mathrm{pH}$ 8.0) for 60 minutes at $0^{\circ} \mathrm{C}$ (Mihashi et al., 1979; Estes et al., 1992; Orlova \& Egelman, 1993; Gershman et al., 1994). The divalent cation will exchange completely under this condition, because the polymerization is much slower (after the 60 minute incubation, only $\sim 3 \%$ of actin was pelletable at $350,000 \mathrm{~g}$ for one hour at $\left.4^{\circ} \mathrm{C}\right)$ than the exchange of divalent cation $(\sim$ one minute at $25^{\circ} \mathrm{C}$; Estes et al., 1992; Gershman et al., 1994). $5 \mu \mathrm{M}$ of this actin was polymerized in the standard solution plus $1 \mathrm{mM}$ EGTA and $1 \mathrm{mM} \mathrm{MgCl}_{2}$ overnight at $4^{\circ} \mathrm{C}$. Immediately before a measurement, labeled $\mathrm{F}_{-} \mathrm{Ca}^{2+}$-actin or F- $\mathrm{Mg}^{2+}$-actin was diluted in buffer A containing $25 \mathrm{mM}$ $\mathrm{KCl}, 4 \mathrm{mM} \mathrm{MgCl}{ }_{2}$ and $25 \mathrm{mM}$ imidazole- $\mathrm{HCl}$ (pH 7.4) to a final concentration of $100 \mathrm{nM}$. Because $\mathrm{Mg}^{2+}$ was in excess of $\mathrm{Ca}^{2+}$ in this solution, weakly binding sites of actin for divalent cations should have been filled with $\mathrm{Mg}^{2+}$ and the only difference between F- $\mathrm{Mg}^{2+}$-actin and $\mathrm{F}-\mathrm{Ca}^{2+}$-actin is considered to be the tightly bound divalent cation.

\section{Beads}

Carboxylated beads $(0.88 \mu \mathrm{m}$ in diameter, Polysciences, Warrington, PA, USA) were covalently coated with $\alpha$-actinin and rhodamine-labeled BSA as follows. Beads were activated with 1\% 1-ethyl-3-(3-(dimethylamino)propyl)carbodiimide in $20 \mathrm{mM}$ sodium phosphate $(\mathrm{pH} 4.5)$ for 3.5 hours at room temperature, and were washed four times with $0.2 \mathrm{M}$ Na-borate $(\mathrm{pH} 8.5)$. To a bead suspension at $1 \%(\mathrm{w} / \mathrm{v}), 0.19 \mathrm{mg} / \mathrm{ml} \alpha$-actinin and $5 \mu \mathrm{g} / \mathrm{ml}$ rhodamine-labeled BSA, which was prepared according to Suzuki et al. (1996), were added and the mixture was incubated for 12 to 15 hours at room temperature. The reaction was quenched by the addition of $10 \mathrm{mM}$ mono-amino-ethanol. The beads were washed four times with Na-borate ( $\mathrm{pH} 8.5$ ) containing $10 \mathrm{mg} / \mathrm{ml}$ BSA. The beads were stored at $4^{\circ} \mathrm{C}$ in a solution containing $20 \mathrm{mM}$ sodium phosphate $(\mathrm{pH} 7.4), 10 \mathrm{mg} / \mathrm{ml}$ BSA, $0.4 \% \quad(\mathrm{w} / \mathrm{v}) \quad \mathrm{NaN}_{3}$ and $5 \% \quad(\mathrm{w} / \mathrm{v})$ glycerol. Immediately before use, they were washed twice with buffer A.

\section{Optical system}

The optical system used in our measurement has been described (Miyata et al., 1995). An infrared microlaser (1053 nm, 1 W, OEM 1053-1000 p, Amoco Laser Co., Naperville, IL, USA) was the light source for the optical 
tweezers. An inverted microscope (Nikon, Diaphot TMD, Tokyo, Japan) with a $100 \times$ objective (oil immersion, numerical aperture $=1.3$; Nikon) was used.

Samples were illuminated simultaneously with a $150 \mathrm{~W}$ halogen lamp (Sigma Koki, PHL-150, Saitama, Japan) and a $100 \mathrm{~W}$ high-pressure mercury lamp (Nikon) for bright field and epifluorescence microscopy. The bright field image was observed with a non-interlace CCD camera (TM-9700, Pulnix, Tokyo, Japan) and the fluorescence image with a CCD camera (CCD-72, Dage-MTI, Michigan, IN, USA) attached to an image intensifier (KS1381, Video Scope International, Sterling, VA, USA). The two images were simultaneously recorded on an $8 \mathrm{~mm}$ video tape (EVO-9650, Sony, Tokyo, Japan) through a multi-viewer (model MV-24C, For A., Tokyo, Japan) and analyzed off-line with a digital image processor (DIPS-C2000, Hamamatsu Photonics, Shizuoka, Japan).

\section{Measurement of the torsional motion of an actin filament}

A flow chamber was constructed of two coverslips (bottom one, $24 \mathrm{~mm} \times 36 \mathrm{~mm}$; top one, $18 \mathrm{~mm} \times$ $18 \mathrm{~mm}$; Matsunami, Osaka, Japan) separated by two spacers of $0.1 \mathrm{~mm}$ thickness. $\alpha$-Actinin-coated beads at $0.04 \%$ were infused into the flow chamber, and were allowed to adhere to the glass surface for five minutes. $\mathrm{BSA} /(2 \mathrm{mg} \mathrm{ml})$ in buffer A was then infused, followed by labeled F-actin at $100 \mathrm{nM}$. After $\sim 15$ minutes when a sufficient number of actin filaments were bound to the surface beads, $0.002 \%$ of beads in buffer B (buffer A plus $20 \mathrm{mM}$ dithiothreitol, $0.20 \mathrm{mg} / \mathrm{ml}$ glucose oxidase, $0.05 \mathrm{mg} / \mathrm{ml}$ catalase, $6.0 \mathrm{mg} / \mathrm{ml}$ glucose and $2 \mathrm{mg} / \mathrm{ml}$ BSA) were introduced. The flow chamber was sealed with nail polish to avoid evaporation. The optical trap was then turned on, and two $\alpha$-actinin-coated beads floating in solution were trapped simultaneously. The two beads aligned vertically in the trap and spontaneously adhered to each other. An actin filament which was bound to two surface beads and which appeared straight between the two beads was selected, and the bead duplex held in the optical trap was manipulated on to the filament. The lower bead was pushed against the filament. After binding, the optical trap was turned off, and the fluctuation of the bead duplex due to the bending and torsional motion of the filament started. The amplitude of the bending motion, however, was small, because we selected those actin filaments that appeared straight between the end beads.

The temperature of the flow chamber was kept at $23( \pm 2)^{\circ} \mathrm{C}$ in all experiments. In some experiments with $\mathrm{F}-\mathrm{Mg}^{2+}$-actin, $1 \mathrm{mM}$ EGTA was added to all solutions to ensure the absence of $\mathrm{Ca}^{2+}$, without significant differences in the experimental results.

In order to distinguish between individual beads in the duplex, the focus of the bright field image was adjusted in such a way that the upper bead appeared bright and the lower bead dark (Figure 1(c) and (d)).

\section{Effect of limited time resolution}

The tilt angle of the bead duplex obtained from the video analysis was the average over $33 \mathrm{~ms}$ (one video frame). The variance of the torsional angles was therefore slightly underestimated. The degree of underestimation is evaluated as follows. The measured angle, $\theta(t)$, is given by:

$$
\theta(t)=(1 / \Delta t) \int_{t}^{t+\Delta \mathrm{t}} \mathrm{d} t^{\prime} \theta^{\prime}\left(t^{\prime}\right)
$$

where $\Delta t=33 \mathrm{~ms}$ and $\theta^{\prime}\left(t^{\prime}\right)$ is the true angle at time $t^{\prime}$. The experimental variance $\left\langle(\theta-\langle\theta\rangle)^{2}\right\rangle$ is related to the true variance $\left\langle\left(\theta^{\prime}-\left\langle\theta^{\prime}\right\rangle\right)^{2}\right\rangle$ by:

$$
\begin{aligned}
\left\langle(\theta-\langle\theta\rangle)^{2}\right\rangle= & \left\langle\left[(1 / \Delta t) \int_{t}^{t+\Delta t} \mathrm{~d} t\left\{\theta^{\prime}\left(t^{\prime}\right)-\left\langle\theta^{\prime}\right\rangle\right\}^{2}\right]\right\rangle \\
= & (1 / \Delta t)^{2} \int_{t}^{t+\Delta t} \int_{t}^{t+\Delta t} \mathrm{~d} t_{1} \mathrm{~d} t_{2} \\
& \times\left\langle\left\{\theta^{\prime}\left(t_{1}\right)-\left\langle\theta^{\prime}\right\rangle\right\}\left\{\theta^{\prime}\left(t_{2}\right)-\left\langle\theta^{\prime}\right\rangle\right\}\right\rangle \\
= & (1 / \Delta t)^{2} \int_{t}^{t+\Delta t} \int_{t}^{t+\Delta t} \mathrm{~d} t_{1} \mathrm{~d} t_{2} \\
& \times\left[\left\langle\left\{\theta^{\prime}-\left\langle\theta^{\prime}\right\rangle\right\}^{2}\right\rangle \exp \left(-\left|t_{1}-t_{2}\right| / \tau\right)\right] \\
= & \left\langle\left\{\theta^{\prime}-\left\langle\theta^{\prime}\right\rangle\right\}^{2}\right\rangle\left[2 \cdot(\tau / \Delta t)^{2}\{(\Delta t / \tau)\right. \\
& -1+\exp (-\Delta t / \tau)\}]
\end{aligned}
$$

The correlation time, $\tau$, was 0.42 seconds for $\mathrm{F}_{-} \mathrm{Ca}^{2+}$-actin

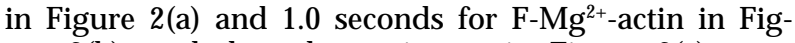
ure 2(b), and thus the variances in Figure 2(c) were underestimated by $3 \%$ and $1 \%$, respectively. The shortest correlation time among all samples in Figure 3 was 0.18 seconds, hence the largest underestimation was $6 \%$.

\section{Estimation of the tension exerted on a filament}

The tension on a filament of which both ends are fixed (on beads) can be estimated from the amplitude $\delta$ of the bending Brownian motion as follows. The work required to achieve a filament conformation $\boldsymbol{u}(y)$, where $\boldsymbol{u}=\left(u_{x}, u_{y}\right)$ is a small deviation away from the straight conformation along the $y$ axis, is given by (MacKintosh et al., 1995):

$$
H=\int_{0}^{\Lambda} \mathrm{d} y\left\{\frac{1}{2} \kappa_{F}\left(\mathrm{~d}^{2} \boldsymbol{u} / \mathrm{d} y^{2}\right)^{2}+1 / 2 F(\mathrm{~d} u / \mathrm{d} y)^{2}\right\}
$$

where $F$ is the tension exerted on the filament and $\Lambda$ is the distance between the fixed ends. The first term represents the bending energy and the second term the work against the tension. The filament conformation can be described by the Fourier series $\boldsymbol{u}(y)=\Sigma_{q} \boldsymbol{u}_{q} \sin (q y)$, where $q=\pi / \Lambda, 2 \pi / \Lambda, \ldots$ are wave vectors. By applying the equipartition principle to the mean square amplitude $\left\langle u_{q}^{2}\right\rangle$, we obtain the bending amplitude at the middle of the filament, $\delta$, in the $x-y$ plane as:

$$
\delta=\left(\left\langle u_{x}(\Lambda / 2)^{2}\right\rangle\right)^{1 / 2}=\left(k_{B} T \Lambda / 4 F\right)^{1 / 2}
$$

Here we have neglected the first term in the integral in equation (7) which turns out to be small for small-amplitude bending. The tension is thus estimated from $\delta$ as:

$$
F=k_{B} T \Lambda / 4 \delta^{2}
$$

The bending amplitude $\delta$ was estimated from the movement of the bead duplex attached to the filament. If the variance of the $x$-excursion of the upper bead is $U_{x}^{2}$ and that of the lower bead $L_{x}^{2}$, the contribution from torsional motion (tilting of the duplex) is, respectively $\left(U_{x}^{2}-\delta^{2}\right)$ and $\left(L_{x}^{2}-\delta^{2}\right)$. For the geometry in Figure 1(a), the ratio $K=\left(U_{x}^{2}-\delta^{2}\right)^{1 / 2} /\left(L_{x}^{2}-\delta^{2}\right)^{1 / 2}$ should be three, as 
already mentioned in Results. $K$ is higher if the filament is wound around the lower bead and thus the center of rotation comes closer to the bead center. In practice, precise determination of $K$ was difficult, but inspection of the filament images indicated that the winding angle was well within $120^{\circ}$ and therefore $K$ was less than five. The bending amplitude in each filament was thus estimated as $\delta=\left\{L_{x}^{2}\left(K^{2}-U_{x}^{2} / L_{x}^{2}\right) /\left(K^{2}-1\right)\right\}^{1 / 2}$ with the uncertainty in $K$ of $3<K<5$. In Figure 3, filaments for which the lower bound of $F(K=5)$ exceeded $1.5 \mathrm{pN}$ are marked with a white arrowhead and those for which the upper bound $(K=3)$ was below $0.3 \mathrm{pN}$ are marked with a black arrowhead.

\section{Measurement of the flexural rigidity of actin filaments}

The flexural rigidity of actin filaments was estimated according to Ott et al. (1993) and Isambert et al. (1995) with slight modification as follows. About $0.5 \mu \mathrm{l}$ of labeled F-actin $(\sim 1 \mathrm{nM})$ in buffer B on a $24 \mathrm{~mm} \times 36 \mathrm{~mm}$ coverslip coated with BSA was spread under a $18 \mathrm{~mm} \times 18 \mathrm{~mm}$ coverslip coated with BSA. Two-dimensional thermal motion of an actin filament in the 1 to $2 \mu \mathrm{m}$ thick chamber was imaged at five second intervals for 80 seconds. The filament images were clipped at a threshold intensity to eliminate the background, resulting in filament images with a thickness $\sim 1 \mu \mathrm{m}$. For each filament image, an arc (radius $1 \mu \mathrm{m}$ ) crossing the filament was generated with the arc center at one end of the filament. The centroid of filament intensity under the arc was calculated, and a next arc was generated with its center at the previous centroid. This process was repeated until the whole filament was covered with dots representing the centroids. Each filament image thus yielded a data set $t(L)$, where $t$ is a unit vector along the $1 \mu \mathrm{m}$ segment connecting two consecutive dots and $L$ is the distance along the filament at intervals of $1 \mu \mathrm{m}$. From many images, CCF was calculated as $\langle\boldsymbol{t}(x) \boldsymbol{t}(x+L)\rangle$ where $x$ is also the distance along a filament.

\section{Acknowledgements}

We thank Mr S. Nishiyama for providing us column purified actin, Drs T. Nishizaka and S. Ishiwata for discussion, and Drs A. Ikegami and Y. Inoue for support. This work was supported by Keio University Special Grant-in-Aid for Innovative Collaborative Research Projects, Grants-in-Aid from the Ministry of Education, Science and Culture of Japan and by Special Coordination Funds for Promoting Science and Technology from the Agency of Science and Technology of Japan. R. Y. is a Research Fellow of the Japan Society for the Promotion of Science.

\section{References}

ben-Avraham, D. \& Tirion, M. M. (1995). Dynamic and elastic properties of F-actin: a normal-modes analysis. Biophys. J. 68, 1231-1245.

Bremer, A., Millonig, R. C., Sutterlin, R., Engel, A., Pollard, T. D. \& Abei, U. (1992). The structural basis for the intrinsic disorder of the actin filament: the "lateral slipping" model. J. Cell. Biol. 115, 689-703.

Egelman, E. H., Francis, N. \& DeRosier, D. J. (1982). F-actin is a helix with a random variable twist. Nature, 298, 131-135.
Egelman, E. H. \& DeRosier, D. J. (1992). Image analysis shows that variations in actin crossover spacings are random, not compensatory. Biophys. J. 63, 1299-1305.

Erickson, H. P. (1989). Co-operativity in protein-protein association: the structure and stability of the actin filament. J. Mol. Biol. 206, 465-474.

Estes, J. E., Selden, L. A., Kinosian, H. J. \& Gershman, L. C. (1992). Tightly-bound divalent cation of actin. J. Muscle Res. Cell Motil. 13, 272-284.

Finer, J. T., Simmons, R. M. \& Spudich, J. A. (1994). Single myosin molecule mechanics: piconewton forces and nanometre steps. Nature, 368, 113-119.

Ford, L. E., Huxley, A. F. \& Simmons, R. M. (1981). The relation between stiffness and filament overlap in stimulated frog muscle fibers. J. Physiol. 311, 219-249.

Gershman, L. C., Selden, L. A., Kinosian, H. J. \& Estes, J. E. (1994). Actin-bound nucleotide/divalent cation interactions. In Actin: Biophysics, Biochemistry, and Cell Biology (Estes, J. E. \& Higgins, P. J., eds), pp. 35-49. Plenum Press, New York.

Gittes, F., Mickey, B., Nettleton, J. \& Howard, J. (1993). Flexural rigidity of microtubules and actin filaments measured from thermal fluctuations in shape. J. Cell Biol. 120, 923-934.

Goldman, A. J., Cox, R. G. \& Brenner, H. (1967). Slow viscous motion of a sphere parallel to a plane wall-I: motion through a quiescent fluid. Chem. Eng. Sci. 22, 637-651.

Holmes, K. C., Popp, D., Gebhard, W. \& Kabsch, W. (1990). Atomic model of the actin filament. Nature, $347,44-49$.

Huxley, A. F. (1957). Muscle structure and theories of contraction. Progr. Biophys. Mol. Biol. 7, 255-318.

Huxley, A. F. \& Simmons, R. M. (1971). Proposed mechanism of force generation in striated muscle. Nature, 232, 533-538.

Huxley, H. E. (1969). Mechanism of muscular contraction. Science, 164, 1356-1366.

Huxley, H. E., Stewart, A., Sosa, H. \& Irving, T. (1994). $X$-ray diffraction measurements of the extensibility of actin and myosin filaments in contracting muscle. Biophys. J. 67, 2411-2421.

Isambert, H., Venier, P., Maggs, A. C., Fattoum, A., Kassab, R., Pantaloni, D. \& Carlier, M.-F. (1995). Flexibility of actin filaments derived from thermal fluctuations. J. Biol. Chem. 270, 11437-11444.

Ishijima, A., Harada, Y., Kojima, H., Funatsu, T., Higuchi, H. \& Yanagida, T. (1994). Single-molecule analysis of the actomyosin motor using nano-manipulation. Biochem. Biophys. Res. Commun. 199, 1057-1063.

Ishiwata, S., Kinosita, K. Jr., Yoshimura, H. \& Ikegami, A. (1987). Rotational motions of myosin heads in myofibril studied by phosphorescence anisotropy decay measurements. J. Biol. Chem. 262, 8314-8317.

Kasai, M. \& Oosawa, F. (1969). Behavior of divalent cations and nucleotides bound to F-actin. Biochim. Biophys. Acta. 172, 300-310.

Kitazawa, T., Shuman, H. \& Somlyo, A. P. (1982). Calcium and magnesium binding to thin and thick filaments in skinned muscle fibers: electron probe analysis. J. Muscle Res. Cell Motil. 3, 437-454.

Kojima, H., Ishijima, A. \& Yanagida, T. (1994). Direct measurement of stiffness of single actin filaments with and without tropomyosin by in vitro nanomanipulation. Proc. Natl Acad. Sci. USA, 91, 1296212966.

Landau, L. D. \& Lifshitz, E. M. (1970). Theory of Elasticity, 2nd English edit., pp. 13-14 and 68-75, Pergamon Press, Oxford. 
MacKintosh, F. C., Käs, J. \& Janmey, P. A. (1995). Elasticity of semiflexible biopolymer networks. Phys. Rev. Letters, 75, 4425-4428.

Mihashi, K., Nakabayashi, M., Yoshimura, H. \& Ohnuma, H. (1979). Absorption, fluorescence, and linear dichroism spectra of fluorescein mercuric acetate (FMA) bound to F-actin. J. Biochem. 85, 359-366.

Miyata, H., Yoshikawa, H., Hakozaki, H., Suzuki, N., Furuno, T., Ikegami, A., Kinosita, K. Jr, Nishizaka, T. \& Ishiwata, S. (1995). Mechanical measurements of single actomyosin motor force. Biophys. J. 68, 286s-290s.

Nishizaka, T., Yagi, T., Tanaka, Y. \& Ishiwata, S. (1993). Right-handed rotation of an actin filament in an in vitro motile system. Nature, 361, 269-271.

Orlova, A. \& Egelman, E. H. (1993). A conformational change in the actin subunit can change the flexibility of the actin filament. J. Mol. Biol. 232, 334-341.

Ott, A., Magnasco, M., Simon, A. \& Libchaber, A. (1993). Measurement of persistence length of polymerized actin using fluorescence microscopy. Phys. Rev. E, 48, R1642-R1645.

Pollard, T. D. \& Cooper, J. A. (1982). Methods to characterize actin filament networks. Methods Enzymol. 85, 211-233.

Prochniewicz, E., Zhang, Q., Howard, E. C. \& Thomas, D. D. (1996). Microsecond rotation dynamics of actin: spectroscopic detection and theoretical simulation. J. Mol. Biol. 255, 446-457.

Ray, S., Meyhöfer, E., Milligan, R. A. \& Howard, J. (1993). Kinesin follows the microtubule's protofilament axis. J. Cell Biol. 121, 1083-1093.

Sawyer, W. H., Woodhouse, A. G., Czarmecki, J. J. \& Blatt, E. (1988). Rotational dynamics of actin. Biochemistry, 27, 7733-7740.
Suzuki, A., Goll, D. E., Singh, I., Allen, R. E., Robson, R. M. \& Stromer, M. H. (1976). Some properties of purified skeletal muscle $\alpha$-actinin. J. Biol. Chem. 251, 6860-6870.

Suzuki, N., Miyata, H., Ishiwata, S. \& Kinosita, K. Jr (1996). Preparation of bead-tailed actin filaments: estimation of the torque produced by the sliding force in an in vitro motility assay. Biophys. J. 70, 401-408.

Tsuda, Y., Yasutake, H., Ishijima, A. \& Yanagida, T. (1996). Torsional rigidity and strength of single actin filaments measured directly by in vitro micromanipulation. Biophys. J. 70, A34.

Vale, R. D. \& Toyoshima, Y. Y. (1988). Rotation and translocation of microtubules in vitro induced by dyneins from Tetrahymena cilia. Cell, 52, $459-469$.

Wakabayashi, K., Sugimoto, Y., Tanaka, H., Ueno, Y., Takezawa, Y. \& Amemiya, Y. (1994). X-ray diffraction evidence for the extensibility of actin and myosin filaments during muscle contraction. Biophys. J. 67, 2422-2435.

Wang, M. C. \& Uhlenbeck, G. E. (1954). On the theory of the Brownian motion II. In Selected Papers on Noise and Stochastic Processes (Wax, N., ed.), pp. 113-132, Dover, New York.

Yanagida, T., Nakase, M., Nishiyama, K. \& Oosawa, F. (1984). Direct observation of motion of single F-actin filaments in the presence of myosin. Nature, 307, 58-60.

Yoshimura, H., Nishio, T., Mihashi, K., Kinosita, K, Jr. \& Ikegami, A. (1984). Torsional motion of eosin-labeled F-actin as detected in the time-resolved anisotropy decay of the probe in the sub-millisecond time range. J. Mol. Biol. 179, 453-467.

Edited by M. F. Moody 